\title{
CAVITY OPTICAL TRANSDUCER PLATFORM WITH INTEGRATED ACTUATION FOR MULTIPLE SENSING APPLICATIONS
}

\author{
T. Michels ${ }^{1 *}$, and V. Aksyuk ${ }^{l}$
}

${ }^{1}$ Center for Nanoscale Science and Technology, National Institute of Standards and Technology, Maryland, USA

\section{ABSTRACT}

We present an on-chip cavity optomechanical transducer platform that combines high measurement bandwidth and very low displacement noise floor with compactness, robustness, small size, and potential for low cost batch fabrication inherent in microelectro- mechanical- systems (MEMS) [1]. Our fiber-pigtailed transducers use surface-micromachined silicon-on-insulator photonic, low-stress silicon nitride structural and metal electrical actuation layers, while front- and backside bulk micromachining defines v-grooves and overhanging cantilevers. The motion of the mechanical devices, such as cantilevers and high mechanical quality factor membrane resonators, is optically measured by integrated silicon micro disk optical cavities. The devices can be actuated electrothermally or electrostatically, and this actuation can also be used to tune readout gain. A displacement noise floor below $10 \mathrm{fm} / \sqrt{ } \mathrm{Hz}$ is achieved for mechanical devices with stiffnesses varying over three orders of magnitude $(\approx 0.2 \mathrm{~N} / \mathrm{m}$ to $\approx 200 \mathrm{~N} / \mathrm{m})$. The combination of electrical actuation, low-loss mechanics, and optomechanical readout will enable a wide variety of high-performance on-chip resonant and non-resonant sensors.

\section{INTRODUCTION}

The measurement of physical quantities by transducing them to a mechanical motion has a long history. The recent advancements in fabrication of micro- and nanomechanical resonators have continued this trend. Ongoing miniaturization and better process control have enabled high quality factors for both optical and mechanical resonators and therefore more sensitive measurement of microscopic physical phenomena. While micromechanical pressure and acceleration sensors are now ubiquitous in consumer electronics and other products of everyday life, in the physics laboratory the micro- and nanoscale resonators allow measurements with unprecedented degree of precision [2].

One of the most significant obstacles to realizing the full potential of micro- and nanomechanical sensing is the readout of the motion of the small resonator with high sensitivity, high bandwidth, and without excess power dissipation. In the past years numerous methods for the readout of resonator motion have been developed [3]. Electrical readout schemes, such as capacitive, magnetomotive, piezoresistive, and piezoelectric, are convenient but suffer from various combinations of poor scaling with reduced size, power dissipation limitations, magnetic field and material requirements, and thermal Johnson noise in the readout signal. On the other hand, optical readout schemes, such as beam deflection and interferometric, substitute optical shot noise for thermal noise, in principle don't dissipate any power at the transducer, and have a high measurement bandwidth. However, to effectively couple motion to light, most of the off-chip optical methods need a certain minimum moving structure size and reflectivity, which often involves bulky structures or mechanically dissipative reflective coatings.

In nanophotonic optical cavities, the light is trapped in a very small volume and is made to interact for a longer timer and more closely with the mechanical resonator. Typical photonic cavity optical quality factors on the order $10^{5}$ to $10^{6}$ increase the readout signal to noise by the same factor. The readout bandwidth is reduced from $\approx 100 \mathrm{THz}$ optical frequency to about $\approx 100 \mathrm{MHz}$, still fast enough for most mechanical sensors. Maintaining stable coupling of a microscopic mechanical resonator with an off-chip optical cavity is challenging due to alignment and drift of components with respect to each other. Here this challenge is overcome by integrating the high quality factor optical cavity directly underneath the moving device, allowing strong interaction with the optical near-field of the cavity, while avoiding mechanical contact (Fig.1). This interaction is described by the optomechanical coupling coefficient $\left(\mathrm{g}_{\mathrm{OM}}\right)$ relating the change in optical frequency of the micro disk cavity to the displacement of the mechanical device. This fully integrated stable and practical optomechanical transducer is fiber connectorized and implements the readout of mechanical motion with gigahertz bandwidth.

Low loss, stable and robust fiber coupling of the transducer is essential to allow sensitive and reliable operation. Therefore, the fibers have to be securely attached to the chip without the introduction of high excess losses between the on-chip waveguide and the optical fiber.

This readout approach allows independent tailoring of the various optical and mechanical parts of the transducer. The photonics can be separately optimized for low losses, high quality factor and desired cavity size, while tuning the waveguide-cavity coupling depth and the optomechanical coupling to achieve the optimal readout sensitivity and dynamic range. The mechanical components' size, shape, stiffness, and resonance frequency can be tailored to best address the specific sensing applications. The actuation can be tailored for the needed displacement and force ranges, ideally without introducing mechanical losses, avoiding increased mechanical noise and decreased $\mathrm{Q}$ in resonators.

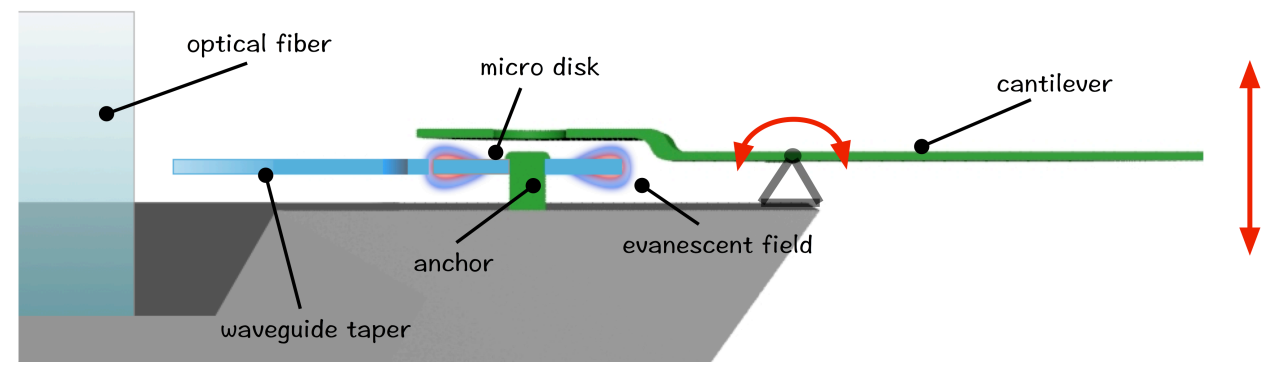

Figure 1: Exemplary schematic of the transducer (not to scale) showing overhung cantilever on a torsional pivot as the mechanical device. SiN is shown in green, Si is shown in blue and grey. The red arrow indicates the direction of movement. 


\section{TRANSDUCER PLATFORM COMPONENTS AND DESIGN}

Figure 1 schematically illustrates the arrangement of the different components in our transducer platform, such as the optical fiber, inverse-taper coupler, waveguides, microdisk cavity, and the mechanical (torsional cantilever) structure. Electricallycontrolled actuators (not shown) are also included in the platform to tune the static position and dynamically excite the motion of the mechanical device. The photonic structures for operation in the telecommunication wavelength range are fabricated in the silicon device layer of a silicon-on-insulator (SOI) wafer, because of the outstanding optical and mechanical properties of silicon. The mechanical device is created in silicon nitride ( $\mathrm{SiN})$, because it shows good mechanical properties resulting in high quality factor devices, has low optical loss, an index of refraction below that of $\mathrm{Si}$ and is compatible with a hydrofluoric (HF) acid release. For the metallization we choose gold with a chromium adhesion layer $(\mathrm{Cr} / \mathrm{Au})$, compatible with $\mathrm{HF}$ and potassium hydroxide $(\mathrm{KOH})$ etches. Furthermore, the combination of $\mathrm{SiN}$ and $\mathrm{Cr} / \mathrm{Au}$ shows a good thermal bimorph actuation efficiency. Silicon dioxide is used as the sacrificial material.

The sensitivity to motion is proportional to the optical quality factor of the micro disk cavity and quickly increases with decreasing gap between the cavity and the mechanical resonator [1]. It is therefore important to accurately locate the mechanical structure in close proximity to an optical micro disk cavity, while maintaining the high optical quality factor of the micro disk optical mode. In our design, the micro resonator is lithographically aligned to the disk, and completely encloses it, while a sacrificial layer defines the gap in the fabrication process. Dedicated lithography and etch steps are used to reduce the sacrificial layer thickness to a predetermined value at the optomechanical transducer, allowing us to control the gap within tens of nanometers, while keeping a thicker silicon dioxide sacrificial and cladding layer elsewhere

Coupling light from optical fiber to an on-chip waveguide may result in high losses due to mode-size and effective-index mismatch between the optical fiber and the $\mathrm{Si}$ waveguide structure, which induce optical scattering and backreflection. Tapering from the waveguide dimensions to the fiber mode dimensions for improving coupling efficiency between optical- fiber and waveguide modes have been suggested [4]. However, to avoid excessive coupling to radiation modes in the taper the required typical taper length must be of the order of a millimeter. Inverse tapers, decreasing the waveguide width at the end accomplish low loss coupling by expanding the waveguide mode to match the fiber mode size. Almeida suggested a micrometer- long nanotaper coupler that converts both the mode size and the effective index of the waveguide to that of the optical fiber [5]. The nanotaper is fabricated in the silicon device layer of a SOI wafer and is butt coupled to the optical fiber. In this coupling scheme, the optical fiber has to be no more than a few micrometer away from the tapered waveguide end. However, it is difficult to fix the optical fiber in the optimum position for the best coupling, without any support structure. Therefore, we etched v-grooves into our chip to be able to actively align and securely attach the optical fibers in the optimum optical coupling position with UV curable epoxy. Since the mode size and effective index of the taper strongly depend on the surroundings of the nanotaper we decided to use an overhanging silicon nanotaper in air, avoiding the possibility of increased losses into the nearby substrate. Simulations were performed to maximize the mode size and effective index overlap and therefore the coupling efficiency. All simulations were performed at $\lambda=1.55 \mu \mathrm{m}$. As input mode reference, a Gaussian beam with the diameter of a single mode optical fiber was used. The waveguide core material was $\mathrm{Si}$ (refractive index of silicon used: $\mathrm{n}_{\mathrm{Si}}=3.48$ ). The waveguide height and width were taken as $\mathrm{h}$ $=260 \mathrm{~nm}$ and $\mathrm{w}_{\mathrm{w}}=500 \mathrm{~nm}$, respectively. The transmission $\left(\mathrm{S}_{21}\right)$ has been calculated for TE- and TM- like modes. A parametric sweep has been performed to find the optimal taper tip width, which gives the best optical transmission and can still be fabricated within the presented process without more than $5 \%$ deviation from the ideal geometry. Fig. 2 shows the results for $\mathrm{S}_{21}$ as a function of taper tip width. The maximum transmission occurs at a taper tip width of around $\approx 100 \mathrm{~nm}$, which is also a reasonable size for the electron beam lithography used to define the waveguide taper. The taper nominal length is $\approx 50 \mu \mathrm{m}$.

Separating photonic and mechanical layers affords flexibility in the design of the mechanical parts to suit specific sensing applications. We designed cantilever structures, torsional structures, and membranes, on chip structures, and overhanging structures, as well as various types of actuation mechanisms. The membrane structures are designed to have a resonance frequency ranging from $\approx 70 \mathrm{kHz}$ up to $\approx 1 \mathrm{MHz}$ - an SEM of these sensors is embedded into the setup in Figure 3. The cantilevers are designed to combine small size with high resonance frequencies, with a range between $\approx 50 \mathrm{kHz}$ and $\approx 4 \mathrm{MHz}$. The integration of an actuator increases the range of possible applications. The builtin static actuation gives the possibility of tuning the transducer gain and measurement range. This is accomplished by changing the static gap size between the mechanical structure and the optical cavity. We decided to develop designs for two actuations schemes, bimorph and electrostatic actuation. Bimorph actuators deliver fast responses and large force. However, the introduction of metal on the mechanical structure creates significant internal losses and therefore reduces the mechanical quality factor drastically. In contrast, electrostatic fringe field actuation doesn't need any metal in contact with the mechanical member, which lets the mechanical member freely oscillate and doesn't affect the mechanical quality factor. However, the maximum forces, as indicated by simulation, are rather small, but still suitable for on-resonance excitation for frequency sensing. Detailed actuator performance is a subject of a future study.

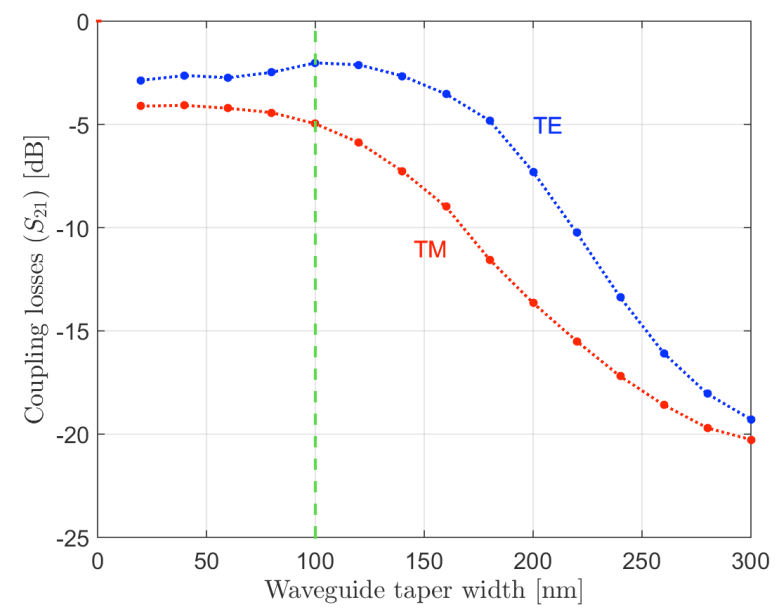

Figure 2: Simulation result of the transmission (S21) as a function of taper tip width. The inverse taper length is $50 \mu \mathrm{m}$. 


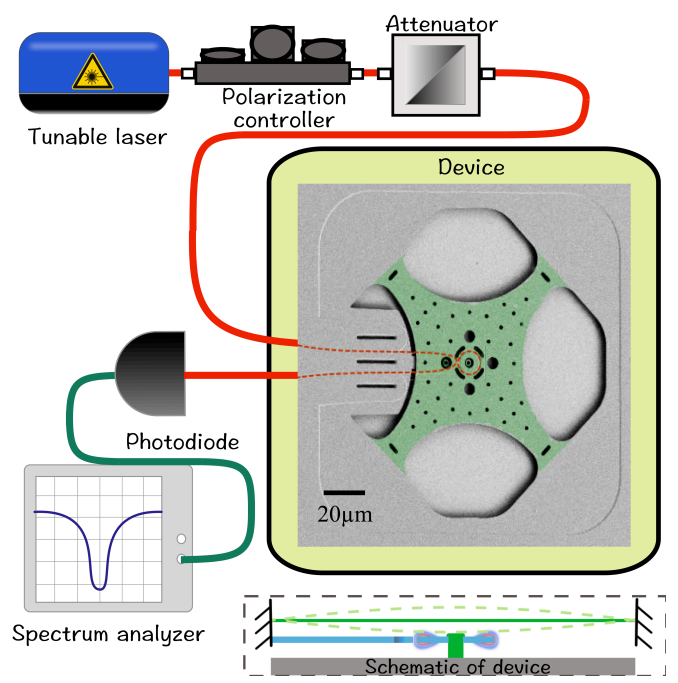

Figure 3: Schematic of the detection setup with an embedded SEM of the membrane transducer and a cross section schematic for this transducer (dashed box).

\section{DEVICE FABRICATION}

The main challenge is to fabricate these diverse optical and mechanical structures in a unified batch fabrication process and a single platform, which can be tailored for specific applications. In the following we will present the process using the overhanging cantilever probe as an example.

The fabrication for the cavity optical transducer is based on double side polished SOI $100 \mathrm{~mm}$ (4”) wafers with a $\approx 260 \mathrm{~nm}$ top silicon layer, with low doping for good optical properties, and a $\approx 2 \mu \mathrm{m}$ buried oxide (BOX) layer. The process flow is summarized in Figure 4. In the first step, the waveguide taper, waveguide, and micro disk are defined via electron beam lithography and inductively-coupled reactive ion etching down to the BOX layer. The nominal width of the waveguide is $\approx 500 \mathrm{~nm}$ and the gap between the waveguide and the disc is defined to be $\approx 340 \mathrm{~nm}$. The waveguide is linearly tapered down to a width of $100 \mathrm{~nm}$ over the distance of $\approx 50 \mu \mathrm{m}$ at both waveguide ends for low loss coupling to/from optical fibers (Fig. 4 b). The remaining structures are defined by i-line stepper optical lithography unless otherwise noted. A sacrificial silicon oxide layer $(\approx 1 \mu \mathrm{m})$ is deposited and defined to create a window to the Si substrate for the later $\mathrm{KOH}$ etching as well as a hole in the center of the micro disk, which is used to anchor the micro disk to the bulk silicon with the following SiN layer. The silicon dioxide is thinned down by a $\mathrm{CF}_{4}$ plasma etch through a lithographically-defined window in photoresist in the region above the micro disk to ensure good optomechanical coupling (Fig. 4 c, d). A low-stress silicon nitride layer $(\approx 400 \mathrm{~nm})$ deposited in a low-pressure chemical-vapor deposition furnace acts as a passivation layer in the waveguide region and as structural material for the mechanical structure. Following nitride deposition, a gold layer is deposited and defined in a liftoff process to create a micro heater, electrical connection, and wire bond pads. (For the electrostatically actuated transducer, the micro heater is replaced by electrodes for fringe field actuation). The SiN layer is lithographically patterned (Fig. 4 e), and dry etched to form the SiN cantilever, SiN ring above the micro disk, and SiN anchor to mechanically attach the micro disk to the bulk silicon. The previously defined metal layer is used as a hard mask for $\mathrm{SiN}$, to self-align the $\mathrm{SiN}$ structure in critical areas (Fig. 4 e). For front side protection during the later $\mathrm{KOH}$ etch, a hafnium oxide (HfO) layer of $\approx 20 \mathrm{~nm}$ is deposited via atomic layer deposition.

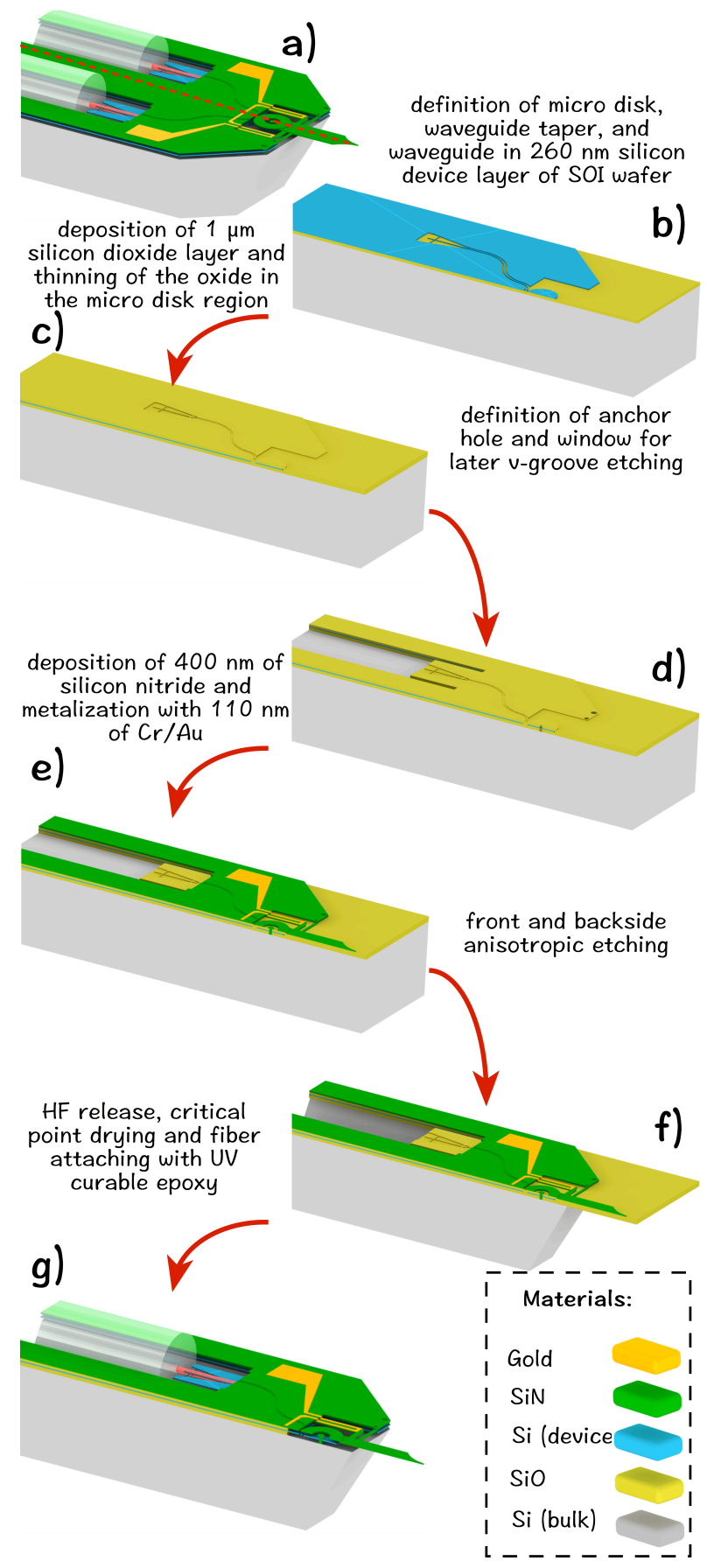

Figure 4: Representation of the process flow for the transducer with integrated thermal actuation and overhanging tip. The image in the top left shows the whole device. The dashed red in indicates the path for the cross sectional views (a).

In the following, a reactive ion etch (RIE) is used to open up a window in the $\mathrm{HfO}$ and $\mathrm{SiN}$ for anisotropic etching of the silicon, to form v-grooves for optical fibers. A back to front aligned backside lithography followed by RIE etching is used to form an anisotropic etch window on the backside as well. Both 
lithographies for the definition of the front and back side etch window for anisotropic etching are defined with contact aligner lithography. During the following anisotropic silicon etch, vgrooves are formed on the front side of the chip and the shape of the cantilever chip is defined by etching through the handle wafer from the backside (Fig. 4 e). (Another approach is the replacement of the backside $\mathrm{KOH}$ etch with a ICP etch to create a backside trench with vertical sidewalls. This approach has been used to develop acceleration sensors with large seismic masses made from the handle wafer.) Silicon dioxide layers and $\mathrm{HfO}$ are removed by $49 \%$ HF wet etching to undercut and release the movable structures as well as the micro disk, which is anchored to the bulk silicon with a SiN anchor. A critical point drying process is used to avoid stiction between the parts due to capillary forces (Fig. $4 \mathrm{f}$ ). At the end of each v-groove the overhanging waveguide inverse tapers are suspended between silicon support structures and coupled to optical fibers, which are placed in the v-groove, actively aligned and glued into place with ultraviolet (UV) light curable epoxy.

\section{MEASUREMENT SETUP}

The detection setup used to characterize the device is shown in Figure 3. Light from a tunable laser $(1520 \mathrm{~nm}$ to $1570 \mathrm{~nm})$ is sent through a polarization controller and coupled into the fiber pigtailed device, allowing for polarization adjustment to maximize the light coupling to the desired micro disk optical mode before recording data. The injected light circulates hundreds or thousands of times (depending on the cavity's finesse) before exiting through the outgoing optical fiber. The output of the fiber is analyzed with a photodetector and either the transmission spectrum of the device is recorded by sweeping the laser wavelength, revealing the spectral location and spectral width of the cavity's optical modes, or modulation of the transmitted intensity as a function of the mechanical motion of the cantilever is measured by fixing the laser wavelength on the shoulder of an optical cavity mode. Motion of the cantilever results in a frequency modulation of the optical cavity modes, which can be translated into an intensity modulation by probing these modes on the side of their resonance minima. The information obtained from the transmission spectra is thus used to determine the laser wavelength for optimal transduction sensitivity. The output signal is intensity-modulated in proportion to the mechanical motion, and is transduced by a photodetector before being sent to an electronic spectrum analyzer to reveal the spectrum of mechanical modes (Fig.5). In electrical actuation experiments a network analyzer is used to measure the device transfer function - a ratio of the optical modulation to the driving force - as a function of the drive frequency.

\section{RESULTS AND DISCUSSION}

Figure 5, shows the measured thermal mechanical noise spectral density of a nitride membrane device held at four corners, similar to the one in Figure 3. A clear peak in the noise spectrum occurs at $\approx 668.1 \mathrm{kHz}$, in good agreement with a finite- element model with a nitride with tensile stress of about $\approx 150 \mathrm{MPa}$. A high mechanical quality factor of better than 110000 is evident from the data.

A TE optical mode with an optical quality factor of $\approx 800000$ was used to carry out this measurement at a very low optical power level of approximately $3.16 \mu \mathrm{W} \quad(-25 \mathrm{dBm})$ excitation power, $830 \mathrm{nW}(-30.8 \mathrm{dBm})$ at the sensor and $219 \mathrm{nW}$ $(-36.6 \mathrm{dBm})$ at the photodetector, accounting for an estimated $5.8 \mathrm{~dB}$ fiber pigtail coupling losses at each facet. Despite the low power, the signal to noise ratio on resonance is approximately 20 dB. Low power was chosen deliberately to demonstrate the sensing performance, while also preventing the optical forces from shifting the frequency of the narrow mechanical resonance, an optical spring effect clearly observed at higher optical powers.

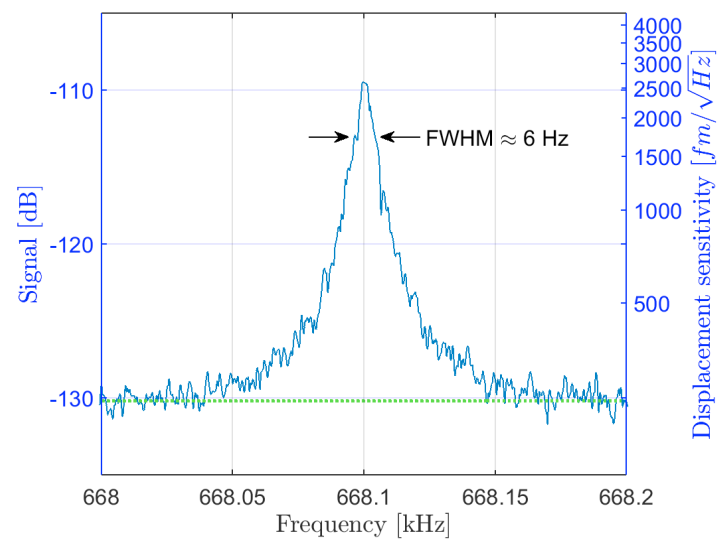

Figure 5: Measured mechanical frequency noise spectrum of the membrane transducer. The dotted green line indicates the background noise level. Signal power is reported relative to $1 \mathrm{~mW}$.

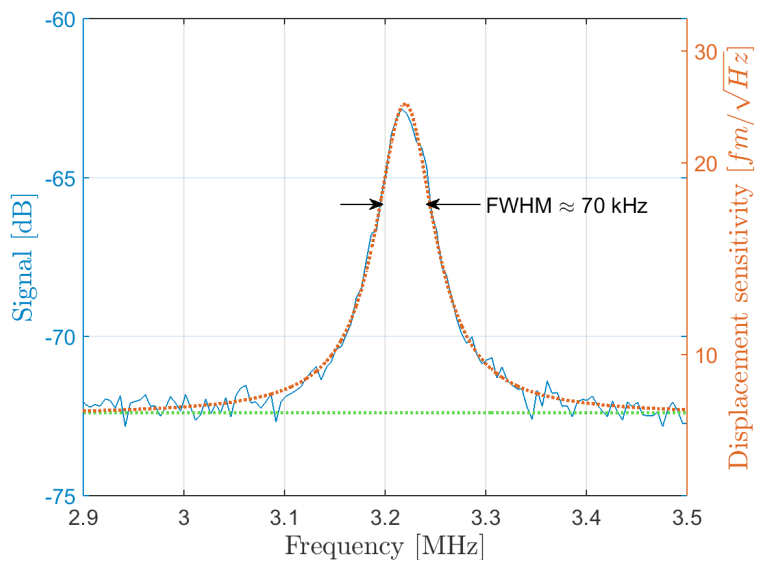

Figure 6: Measured mechanical frequency noise spectrum of the cantilever transducer with Lorentzian fit. The dotted green line indicates the background noise level. Signal power is reported relative to $1 \mathrm{~mW}$.

Figure 6, shows the measured thermal mechanical noise power spectral density of a torsional cantilever transducer. The red line shows a Lorentzian fit of the power spectral density in cantilever displacement, calibrated using the equipartition method [6]. The background corresponds to the measurement noise of $9 \mathrm{fm} / \sqrt{\mathrm{Hz}} \pm 0.5 \mathrm{fm} / \sqrt{\mathrm{Hz}}$. The uncertainty is one standard deviation. The statistical uncertainty derived from the measurement is small. The main uncertainty is propagated form the Young's modulus used to calculate the spring constant for the displacement sensitivity calculation with the equipartition theorem. The estimated variation of the Young's modulus originating from deposition conditions is about $10 \%$.

We measured mechanical quality factors between $\approx 50,000$ and $\approx 500000$ for the low-stress silicon nitride membrane transducers and $\approx 50$ to $\approx 2000$ for the cantilever transducers which include the metallization for bimorph actuators.

Static and dynamic electrothermal actuation has been characterized. First, the static displacement of the silicon nitride 
structure was characterized as a function of voltage with a white light interferometer (Figure 7). The dashed red line shows a $2^{\text {nd }}$ order polynomial fit to indicate the expected displacement/actuation voltage relation.

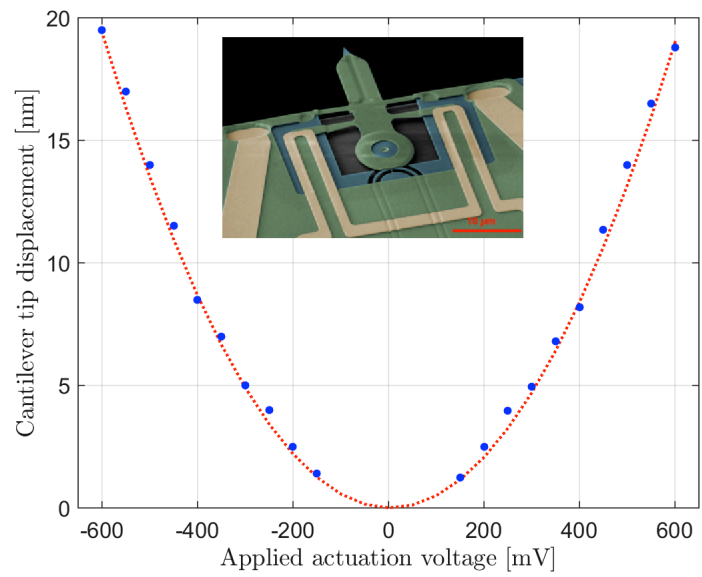

Figure 7: Relative cantilever displacement with applied DC voltage. The statistical uncertainty based on repeated measurements is smaller than the data markers.

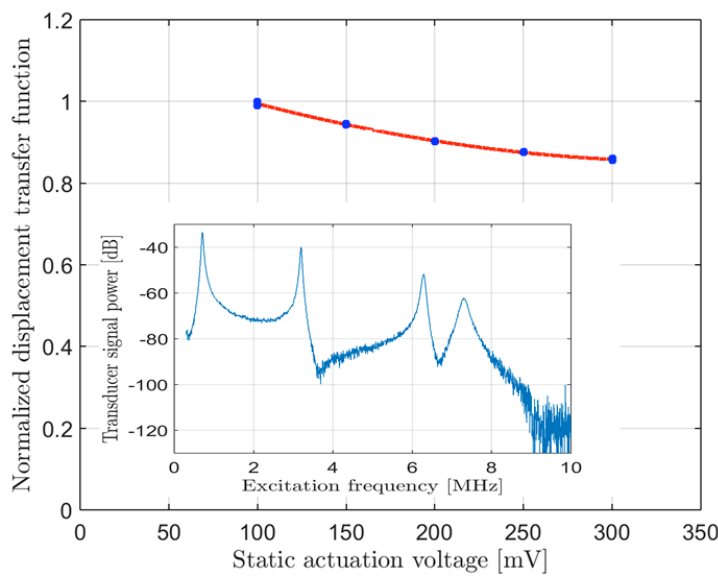

Figure 8: Normalized gain of the optomechanical displacement sensor is decreased by static actuation. The one standard deviation uncertainty based on fitting network analyzer spectrum data is smaller than the data marker size. Inset: Optomechanical signal power as a function of frequency for $8 \mathrm{mVAC}$ and $50 \mathrm{mVDC}$. Signal power is reported relative to $1 \mathrm{~mW}$ (inset).

We then optomechanically measured the dynamic response of the structure to actuation. A small fixed modulation (AC) voltage, swept between $300 \mathrm{kHz}$ and $10 \mathrm{MHz}$, was added to the actuator static bias (DC) voltage, resulting in a small, known mechanical modulation of the gap. The laser wavelength has been fixed at the steepest slope on the side of the optical resonance line at each applied DC voltage. A network analyzer was used to provide the $\mathrm{AC}$ voltage and detect the resulting optical power modulation, which is proportional to the motion amplitude and the optomechanical coupling. A typical optomechanically measured device transfer function is shown on the Figure 8 inset.

The AC bimorph output is proportional to the product of AC and DC voltages, as the actuation force is quadratic in applied voltage. To illustrate the tuning of the optomechanical gain by the actuator, we first normalized the displacement spectra by the DC voltage to account for the stronger drive with larger DC voltage The resulting normalized displacement transfer function (Figure 8) reveals the gain decreasing with increasing bias as the actuator increases the transducer gap and decreases the gain of the optomechanical sensor.

The results show that the small actuation here is capable of tuning the gain by more than $10 \%$.

\section{SUMMARY AND CONCLUSIONS}

In summary, we have presented an overview of the design, micro- and nanofabrication, and characterization of a novel type of fully-integrated cavity optomechanical transducer platform for measurement of physical quantities by transducing them to mechanical motion. The approach of full silicon integration of all nanophotonic components with mechanically separated highquality-factor movable components creates the opportunity to independently tailor optical, mechanical, and the actuation parts for a variety of MEMS and NEMS sensing applications that require high precision, high bandwidth, and small footprint. Additional benefits of the photonic readout approach are low power dissipation at the sensor, insensitivity to electromagnetic interference and robustness of fiber-connectorized devices. We demonstrate high mechanical and optical performance of platform components by optically detecting thermomechanical fluctuations and actuated motion of mechanical devices while tuning the optical readout gain by electrical actuation.

\section{ACKNOWLEDGMENTS}

The author would like to specially thanks Prof. Rangelow, D. Westly, and R. Ilic for helpful discussions during the development. The layout was designed with the "Nanolithography Toolbox", a free software package developed by the Center for Nanoscale Science and Technology.

\section{REFERENCES}

[1] H. Miao, K. Srinivasan, and V. Aksyuk, "A microelectromechanically controlled cavity optomechanical sensing system," New J. Phys., vol. 14, no. 7, p. 075015, Jul. 2012.

[2] J. D. Teufel, T. Donner, M. A. Castellanos-Beltran, J. W. Harlow, and K. W. Lehnert, "Nanomechanical motion measured with an imprecision below that at the standard quantum limit," Nature Nanotech, vol. 4, no. 12, pp. 820 823, Nov. 2009.

[3] T. Michels and I. W. Rangelow, "Review on Scanning Probe Micromachining and its Applications within Nanoscience," Vac. Sci. and Technol., pp. 1-21, Mar. 2014.

[4] I. Moerman, P. P. Van Daele, and P. M. Demeester, “A review on fabrication technologies for the monolithic integration of tapers with III-V smiconductor devices," IEEE J. Select. Topics Quantum Electron., vol. 6, 1997.

[5] V. R. Almeida, R. Panepucci, and M. Lipson, "Nanotaper for compact mode conversion," Optical Letters, pp. 1-3, Jun. 2003.

[6] H. Li, Y. Chen, J. Noh, S. Tadesse, and M. Li, "Multichannel cavity optomechanics for all-optical amplification of radio frequency signals," Nature Communications, vol. 3, pp. 1091-6, 1AD.

\section{CONTACT}

*T. Michels, tel: +1-301-975-2273; Thomas.Michels@nist.gov 